\title{
Tröger, Mandy: Pressefrühling und Profit. Wie westdeutsche Verlage 1989/1889 den Osten eroberten
}

\author{
Herbert von Halem Verlag. 356 Seiten. Preis: $€ 25$
}

\author{
Klaus Beck
}

Online publiziert: 12. Juni 2020

(C) Der/die Autor(en) 2020

Mandy Tröger hat eine bemerkenswerte und von der DGPuK ausgezeichnete Studie zu einer entscheidenden Phase der jüngeren deutschen Pressegeschichte vorgelegt. Sehr deutlich positioniert sich die Autorin als Ostdeutsche und verortet sich in der Kritischen Kommunikationswissenschaft. Bislang überhörte Stimmen ostdeutscher Akteure sollen zu Gehör gebracht und vor dem ,Erfahrungshorizont vieler Ostdeutscher“ (S. 19) Teile der Geschichte umgeschrieben werden. Tröger möchte belegen, dass ,,[n]icht die Unfähigkeit der DDR-Presse zur Anpassung an die neuen Gegebenheiten der Marktwirtschaft“ zu ihrem Untergang oder Verkauf führte, ,sondern die aggressiven Marktstrategien westdeutscher Verlage auf allen Ebenen des DDRPressesektors (Verkauf, Eigentum und Vertriebsstrukturen)“ (S. 19). Der kapitalismus-(kritisch) sozialisierte West-Rezensent fragt sich, worin genau der Widerspruch bestehen soll, denn aggressive Marktstrategien zählen spätestens seit 1949 zu den Gegebenheiten kapitalistischer Pressemärkte. Eine politökonomische Note enthält die These, dass ,die politischen Interessen der damaligen Bundesregierung ... eng gekoppelt an die Wirtschaftsinteressen westdeutscher Verlagsgruppen" waren und das Bundesinnenministerium (BMI) der ausschlaggebende Akteur. Die Regierung Kohl habe politisch profitiert, denn mithilfe der Westpresse konnte sie ,die DDRWahlen maßgeblich mitbestimmen und die Einheitsagenda ... im Osten verbreiten“ (S. 83). Das ist eine starke Medienwirkungsthese, lässt den Rundfunk außen vor und unterstellt eine hochgradig konsonante Berichterstattung der Westmedien. Die Vorstellung, dass Verlage und alle westdeutschen Journalisten aus dem Kanzleramt ferngesteuert Propaganda verbreiten, verwundert in einer kritischen kommunikationswissenschaftlichen Dissertation.

Prof. Dr. K. Beck $(\bowtie)$

Lehrstuhl für Kommunikationswissenschaft, Universität Greifswald,

Ernst-Lohmeyer-Platz 3, 17489 Greifswald, Deutschland

E-Mail: klaus.beck@uni-greifswald.de 
Spannend wie ein „Wirtschaftskrimi“ und mit klar verteilten Täter- und Opferrollen wird ein Stück Pressegeschichte erzählt. Das bietet viele interessante Einblicke und eine echte Bereicherung. Allerdings überzeugen mich nicht alle Interpretationen, denn an einigen Stellen vermisse ich methodisch-systematische Analysen und differenziertere Erklärungen. Die Neuordnung der Presse in der DDR ist vor allem, aber eben nicht nur, eine Ost-West-Geschichte. Die plakative Gegenüberstellung von bürgerbewegtem demokratischen Pressefrühling und kapitalistischer Profitmaximierung verkürzt die detailliert rekonstruierte Komplexität der Aushandlungsprozesse und Vielfalt von Akteuren. Die strukturellen Ergebnisse der Entwicklung sind bekannt und wenig erfreulich, vor allem dort, wo das Westmodell ausnahmsweise nicht übergestülpt wurde (verlagsabhängiges Pressegrosso, Regionalzeitungsstruktur). Daher überzeugt die These, das BMI habe ,den BRD-Standard zum DDRReformhorizont" gemacht (S. 192) weniger als die nur angedeutete Einordnung in den mediensystematischen und -ökonomischen Kontext. Die kapitalkräftigen Großverlage haben jahrzehntelang versucht, das der „Netzneutralität“ verpflichtete mittelständische Pressegrosso auszuschalten, um ihre Größenvorteile gegenüber kleineren Verlagen zu monetarisieren; die DDR war hier tatsächlich Experimentierfeld.

Trögers detaillierte Rekonstruktion lässt erkennen, dass die Geschichte vielschichtiger ist: Die sehr frühzeitig auf Marktexpansion drängenden Westverlage stoßen nicht nur bei den formal für die DDR nicht zuständigen bundesdeutschen Politikern auf keinerlei Widerstand, sie finden in der DDR-Politik „Verbündete“ mit partiell ähnlichen Interessen. Vor allem treffen sie auf schwache Institutionen, die vor riesigen, unter enormem Zeitdruck zu lösenden Problemen stehen. Die DDR-Post besaß mit dem Pressevertrieb (PVZ) eine Monopolstellung, die sich als dysfunktional erwies: Weder die neuen Ost-Zeitungen noch die importierten Westprodukte wurden zuverlässig genug vertrieben, um die Informationsfreiheit in der DDR (und weniger die Medienfreiheit) zu gewährleisten. Die großen Westverlage ergriffen deshalb die Initiative für eine eigene Vertriebsstruktur, die Tröger als entscheidende „Hintertür" für die Markteroberung bezeichnet und der sie rund 160 Seiten widmet. Diese Fokussierung auf den Pressevertrieb passt sehr gut in das „Wildwest im Osten"-Narrativ, blendet aber die anderen ökonomischen Ursachen aus: Papiermangel, technische Qualitäts- und Layoutmängel, Probleme im neuen Feld der Werbeakquise sowie Wegfall der Subventionen und Dumpingpreise der Westkonkurrenz werden in den Dokumenten und Zeitzeugnissen sowie vom Medienkontrollrat und dem Amt für Wettbewerbsschutz der DDR aufgeführt, aber nicht zur Erklärung genutzt. Das medienökonomische Kernproblem lag im Valuta- und Kapitalmangel der DDR-Betriebe, insbesondere der Neugründungen. Vieles spricht dafür, dass es auch bei einem neutralen und leistungsfähigen Vertrieb zu einem ungleichen oder gar unfairen Wettbewerb gekommen wäre. Stärkere DDR-Institutionen vorausgesetzt, hätte staatliche Regulierung in Gestalt von Importbeschränkungen für Westmedien den Binnenmarkt zwar geschützt. Den Idealen der Bürgerbewegung, dem Medienbeschluss der Volkskammer und dem Völkerrecht aber hätte eine staatliche Vertriebskontrolle widersprochen.

Für Tröger scheinen allein Westakteure ausschlaggebend: Das BMI stand in „Kontakt ... mit westdeutschen Wirtschaftsgruppen“(S. 98), sie ,untergruben ... schließlich staatliche Regulierungsversuche der Noch-DDR“ (S. 105). Dass die Westverlage di- 
rekt nach dem Mauerfall initiativ wurden, spricht dafür, dass es einer Abstimmung oder stillschweigenden Duldung seitens des BMI gar nicht bedurfte. Dass sich die Westverlage wenig für die Chancen der Ostverlage interessiert haben, verwundert weniger als dass die DDR-Post die Interessen der neuen DDR-Presse konterkarierte. Es zeigt aber, dass die Ost-West-Geschichte nur ein Teil der Wahrheit ist, die Konfliktlinien jedoch komplexer verlaufen und das faktische Zusammenwirken von Ostund Westakteuren betreffen. Die Verfasserin räumt ein, dass die ,neu gewählte DDRRegierung“ die marktliberale Überzeugung der Bundesregierung und der Verlegerbände teilte, unterstellt aber ein Problembewusstsein, das sich in der praktischen Politik nicht nachweisen lässt.

Pauschal sehr gut weg kommt die DDR-Presse, denn es ,... hatten alle Verlage, Zeitungen, Redakteure, Journalisten und Leser versucht, neue Standpunkte zu gesellschaftspolitischen und praktischen Fragen zu finden ... eine freie Presse neu definieren, praktisch und nicht als rhetorisches Mittel“ (S. 279). Dass die Rede vom „Anschluss der DDR“ (S. 273) ein historisch gelungener Vergleich ist, bezweifele ich. Eine systematischere Analyse der Akteursnetzwerke, Interessenkollisionen und -koalitionen, der widersprüchlichen Kapitalinteressen und -strategien könnte helfen, besser zu verstehen, warum die Bedürfnisse der neuen DDR-Presse ignoriert und warum vernünftige und berechtigte medienpolitische Forderungen im DDRGefüge ,verhallten“ (S. 75). Der Feststellung, dass (auch) hinsichtlich der Medienordnung die Chance verpasst wurde, nach besseren Lösungen zu suchen als sie in der Bundesrepublik bis heute unverändert praktiziert werden, kann man zweifellos zustimmen.

Funding Open Access funding provided by Projekt DEAL.

Open Access Dieser Artikel wird unter der Creative Commons Namensnennung 4.0 International Lizenz veröffentlicht, welche die Nutzung, Vervielfältigung, Bearbeitung, Verbreitung und Wiedergabe in jeglichem Medium und Format erlaubt, sofern Sie den/die ursprünglichen Autor(en) und die Quelle ordnungsgemäß nennen, einen Link zur Creative Commons Lizenz beifügen und angeben, ob Änderungen vorgenommen wurden.

Die in diesem Artikel enthaltenen Bilder und sonstiges Drittmaterial unterliegen ebenfalls der genannten Creative Commons Lizenz, sofern sich aus der Abbildungslegende nichts anderes ergibt. Sofern das betreffende Material nicht unter der genannten Creative Commons Lizenz steht und die betreffende Handlung nicht nach gesetzlichen Vorschriften erlaubt ist, ist für die oben aufgeführten Weiterverwendungen des Materials die Einwilligung des jeweiligen Rechteinhabers einzuholen.

Weitere Details zur Lizenz entnehmen Sie bitte der Lizenzinformation auf http://creativecommons.org/ licenses/by/4.0/deed.de.

Prof. Dr. Klaus Beck ist Inhaber des Lehrstuhls für Kommunikationswissenschaft der Universität Greifswald. 